\title{
Is diagnosing metabolic syndrome a uniquely simple way to predict incident type 2 diabetes mellitus?
}

\author{
Gerald M. Reaven MD
}

$\infty$ See related research paper by Ley and colleagues, page 617

$\mathrm{T}$

he perceived need for introducing metabolic syndrome as a diagnostic category was to identify individuals at increased risk of cardiovascular disease whose primary abnormality is not an elevated low-density lipoprotein cholesterol level. ${ }^{1,2}$ Identification of such individuals would permit initiation of lifestyle interventions to decrease their risk of cardiovascular disease. Much has been published since the appearance of specific definitions of metabolic syndrome, and use of the syndrome has been extended beyond the original intent. However, questions have been raised concerning the clinical utility of labelling someone as having metabolic syndrome.

The 2 most commonly cited definitions of metabolic syndrome are those of the National Cholesterol Education Program $^{1}$ and the International Diabetes Federation. ${ }^{2}$ Whereas the 2 definitions differ substantially as to the diagnostic criteria for metabolic syndrome, both include the same 5 components: increased adiposity; hypertension; some degree of hyperglycemia; a high triglyceride level; and a low level of highdensity lipoprotein cholesterol. Meeting the dichotomous cut-off points for an abnormality in 3 or more of the 5 components fulfills the requirements for diagnosis according to the definition of the National Cholesterol Education Program. In the case of the definition of the International Diabetes Federation, the diagnosis is based on ethnic-specific values for abdominal obesity plus any 2 of the other 4 components.

In this context, it is interesting that an analysis of data from the Framingham study ${ }^{3}$ demonstrated no substantial increase in risk associated with clusters of 3 traits compared with clusters of 2 traits. Also, data from the Third National Health and Nutrition Examination Survey ${ }^{4}$ indicated that each of the 5 components of metabolic syndrome, with the possible exception of obesity, was an independent predictor of cardiovascular disease. Therefore, it is not surprising that controversy exists over whether a diagnosis of metabolic syndrome provides more useful information about cardiovascular disease risk than its individual components do.

Although not initially intended for this purpose, investigators have used metabolic syndrome to identify individuals at increased risk of type 2 diabetes mellitus. A review of studies published from 1998 to $2008^{5}$ concluded that "metabolic syndrome, however defined, has a stronger association with incident diabetes than that previously demonstrated for coronary heart disease." This conclusion is consistent with what is known about the pathogenesis of type 2 diabetes. Prospective studies have shown that hyperglycemia occurs when insulinresistant individuals can no longer secrete enough insulin to maintain normal glucose tolerance. ${ }^{6.7}$ None of the components

\section{Key points}

- The 2 commonly cited definitions of metabolic syndrome differ in diagnostic criteria but include the same 5 components: increased adiposity, hypertension, some degree of hyperglycemia, a high triglyceride level and a low level of high-density lipoprotein cholesterol.

- Irrespective of the definition used, metabolic syndrome was as effective as impaired glucose tolerance in predicting incident type 2 diabetes mellitus among Aboriginal Canadians in a 10-year follow-up study.

- The individual components of metabolic syndrome appeared to be comparable to the overall syndrome in predicting incident diabetes.

- Determining a diagnosis of metabolic syndrome may not be the simplest means of identifying a person's risk of diabetes.

of the various definitions of metabolic syndrome are associated with insulin secretory function, but they are all related to insulin resistance. ${ }^{8}$

The fundamental question to consider is not whether metabolic syndrome can identify individuals at risk of type 2 diabetes - it certainly can - but whether its use for this purpose offers any unique clinical benefit. In this issue of CMAJ, Ley and colleagues ${ }^{9}$ conclude that such is the case among Aboriginal Canadians. In particular, they conclude that metabolic syndrome, defined using readily available clinical variables, may be useful for identifying individuals at risk of type 2 diabetes in remote Aboriginal communities, where 2-hour oral glucose tolerance tests to determine impaired glucose tolerance are not easily applied.

Being able to identify patients at increased risk of diabetes effectively without the need to measure plasma glucose levels after an oral glucose load is of obvious clinical utility. However, Lorenzo and colleagues ${ }^{10}$ found that, in a study involving mostly Hispanic Americans, impaired glucose tolerance had a higher positive predictive value than each of the components of metabolic syndrome. Thus, the results reported by Ley and colleagues may not be true of all population groups.

More importantly, although Ley and colleagues emphasize the relative similarity of metabolic syndrome and impaired glucose tolerance in identifying individuals at risk of diabetes in a nondiabetic population, they seem to have done so at the expense of other measures that might be of equal and simpler clinical utility. The authors' report that the individual components of metabolic syndrome were generally comparable to

Gerald Reaven is with Division of Cardiovascular Medicine, Stanford University School of Medicine, Stanford, USA. 
the syndrome overall in terms of sensitivity, specificity, and positive and negative predictive values. Similarly, blood pressure and triglyceride and fasting plasma glucose levels were all found to be statistically significant predictors of incident diabetes. The authors' comparison of C statistics seems to suggest that the predictive value of metabolic syndrome is not particularly different from that of its individual components.

In conclusion, Ley and associates have shown, as have others, ${ }^{3,10}$ that metabolic syndrome can identify individuals at risk of type 2 diabetes. Whether it provides a uniquely simple way to accomplish the task is not as certain. It might have been useful if the authors had focused less on the comparison of the clinical utility of metabolic syndrome relative to that of impaired glucose tolerance and more on the comparison of the diagnostic utility of metabolic syndrome relative to that of its individual components. It is possible that some combination of 2 of the 5 components (e.g., obesity and a fasting plasma glucose level of $5.6 \mathrm{mmol} / \mathrm{L}$ or higher) may provide an equally effective and simpler approach to identifying a person's risk of diabetes. Nevertheless, the absence of such information should not obscure the fact that Ley and colleagues have shown, in an Aboriginal Canadian population, that abnormalities associated with insulin resistance constitute a prediabetic state.

Competing interests: None declared.

\section{REFERENCES}

1. Expert Panel on Detection, Evaluation, and Treatment of High Blood Cholesterol in Adults. Executive Summary of The Third Report of The National Cholesterol Education Program (NCEP) Expert Panel on Detection, Evaluation, And Treatment of High Blood Cholesterol In Adults (Adult Treatment Panel III). JAMA 2001;285:2486-97.

2. Alberti KG, Zimmet P, Shaw J; IDF Epidemiology Task Force Consensus Group. The metabolic syndrome - a new worldwide definition. Lancet 2005;366:1059-62.

3. Wilson PW, D'Agostino RB, Parise H, et al. Metabolic syndrome as a precursor of cardiovascular disease and type 2 diabetes mellitus. Circulation 2005;112:3066-72.

4. Ninomiya JK, L'Italien G, Criqui MH, et al. Association of the metabolic syndrome with history of myocardial infarction and stroke in the Third National Health and Nutrition Examination Survey. Circulation 2004;109:42-6.

5. Ford ES, Li C, Sattar N. Metabolic syndrome and incident diabetes: current state of the evidence. Diabetes Care 2008;31:1898-904.

6. Warram JH, Martin BC, Krolewski AS, et al. Slow glucose removal rate and hyperinsulinemia precede the development of type II diabetes in the offspring of diabetic parents. Ann Intern Med 1990;113:909-15.

7. Lillioja S, Mott DM, Spraul M, et al. Insulin resistance and insulin secretory dysfunction as precursors of non-insulin-dependent diabetes mellitus. Prospective studies of Pima Indians. N Engl J Med 1993;329:1988-92.

8. Reaven GM. Banting lecture 1988. Role of insulin resistance in human disease. Diabetes 1988;37:1595-607.

9. Ley SH, Harris SB, Mamakeesick M, et al. Metabolic syndrome and its components as predictors of incident type 2 diabetes mellitus in an Aboriginal community. CMAJ 2009;180:617-24.

10. Lorenzo C, Okoloise M, Williams K, et al. The metabolic syndrome as a predictor of type 2 diabetes: the San Antonio Heart Study. Diabetes Care 2003;26:3153-9.

Correspondence to: Dr. Gerald M. Reaven, Division of

Cardiovascular Medicine, Stanford University Medical Center,

300 Pasteur Dr., Stanford CA 94305, USA; fax 650 725-1599;

greaven@cvmed.stanford.edu 